\title{
Analysis of Pulse Code Modulation Formats in High Speed Optical Transmission System Using FBG and EDFA.
}

\author{
Navneet Kumar ${ }^{1}$, A.K Jaiswal' ${ }^{2}$, Mukesh Kumar ${ }^{3}$, Anil Kumar ${ }^{4}$, \\ Kri. Neha suman ${ }^{5}$ \\ ${ }^{1}$ (M.Tech Scholar,Dept. Of Ece/Shiats,India), ${ }^{2}$ (Prof. \& H.O.D,Dept. Of Ece/Shiats, India) $)^{3,4}$ (Assistant \\ Prof.,Dept. Of Ece/Shiats,India), ${ }^{5}$ (B.Tech Student,Dept. Of Eee/Shiats,India).
}

\begin{abstract}
The basic barricade to high-speed communication systems and long distance transmission over the installed standard single mode fiber at $1.55 \mu \mathrm{m}$ is the linear chromatic dispersion and nonlinearities. Both parameters have been of great concern as these parameters confine the overall efficiency of the system.FBG are being used as dispersion compensator and EDFA is used for amplification of the signal. The objective of this paper is to analyze the performance of two different modulation schemes i.e. NRZ and RZ modulation format at $10 \mathrm{~GB} / \mathrm{s.NRZ}$ and RZ modulation format is the scheme used to avoid intersymbol interference (ISI) on an optical carrier wave for transmission over optical fiber. Each modulation method has its own advantages and disadvantages for the particular channel conditions. The performance of $N R Z$ and $R Z$ in optical communication system with single channel over single mode fiber is investigated by simulation. Then, based on modulated outputs of RZ and NRZ codes, a comprehensive comparison is developed in terms of $Q$ factor, noise figure, spectrum, eye diagrams and average input power to establish the merits and demerits of the $R Z$ and NRZ codes formats in short as well as long haul optical communication system.
\end{abstract}

Keywords: Opti-system simulator, optical transmission system, Non-return to zero, Return to zero, Q-factor.

\section{Introduction}

Optical communications is a novel technology that offers special features and merits over conventional electrical communication. It provides high speed data rate and enormous capacity. Optical transmission system consists of transmitter part, transmission channel and receiver part [1].FBG is major component used as a dispersion compensator in optical fiber communication system[2].Another key component is EDFA .These provide high bit rate data transmission over long distance with appropriate optical amplification. [3] There are two typical choices for the modulation format of the signal known as the return-to-zero (RZ) and non-return-tozero (NRZ).This paper deals with these formats explore the merits and demerits of NRZ and RZ systems for optimized optical communication system. In this paper, the simulation of the optical transmission system in optical fiber has been discussed by analyzing the different modulation format by using different parameters setting. The value of different parameters has been investigated such as gain $(\mathrm{db})$, noise figure $(\mathrm{db})$,output power and $\mathrm{Q}$ factor.

Opti-System is a comprehensive software design suite that enables users to plan, test, and simulate optical links in the transmission layer of modern optical networks. It is an innovative optical communication system simulation package which was explored by opti Wave Company in order to meet the academic requirement of the system designers, optical communications engineers, researchers [4].

\section{A. Dispersion in fiber and its compensation by FBG}

\section{Optical Impairment Overview}

When a photosensitive fiber is placed in closed proximity to the phase mask,permanent increase in the RI in the fiber core can be achieved. RI increase of the order of $10^{-3}$ is possible. A fiber can be made photosensitive by doping the core with germanium, phosphrous or boron doping during fabrication of preform.Fiber grating selectively transmit some wavelength and reflects others, depending on the spacing of the pattern and RI of glass. A grating will reflects light with a wavelength corresponding to twice the grating period $\Lambda$ multiplied by the effective refractive index of refraction $\eta_{\text {eff }}$. This is called Bragg condition [2]. Light at other wavelength will be transmitted without significant attenuation. In fact, grating operates as a narrow band filter. By using a single wideband source and distributing it to many different gratings, each with a different fringe spacing and hence reflection wavelength, it is possible to generate signals of different wavelengths.

The basic principle of operation of a chirped fiber grating as a dispersion- compensation element is shown in figure 1. Different wavelength components of a broadband pulse are reflecting at different location along the grating resulting in a differential group delay. The maximum delays are proportional to the length of the grating. 
Thus we find FBG dispersion compensator may have wider application in long haul optical fiber communication system.

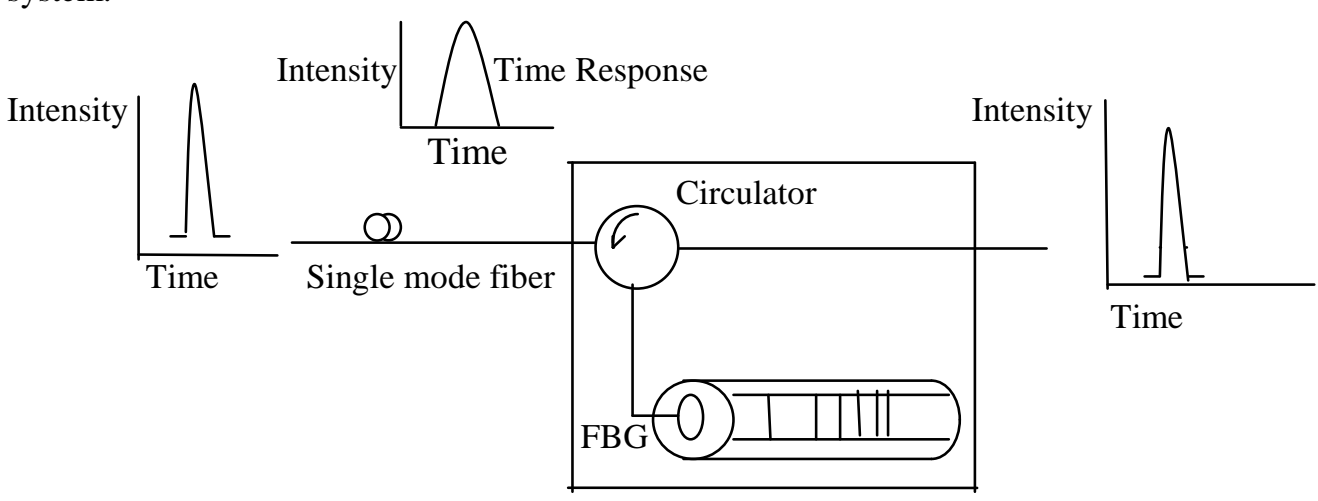

Fig. 1 Basic Principle of operation of chirped fiber grating.

\section{B. Nonlinearities in optical fiber system}

Nonlinear effects in optical fibers are due to either changes in the refractive index of the medium with optical power or scattering phenomenon [7].

(i) Power dependence of the RI is responsible for the Kerr effect. Depending upon the type of input signal, the Kerr nonlinearity manifest itself in different effects such as self phase modulation (SPM), cross phase modulation (XPM) and four wave mixing (FWM).

(ii) Scattering phenomenon are responsible for Brillouin and Raman effects. At high power levels, these can induce stimulated effects and the intensity of the scattered light grows exponentially once the incident power exceeds a threshold level.

In contrast to Rayleigh scattering where the scattered wave has same frequency, non linear scattering process (SRS and SBS) are inelastic which does not only result in frequency shift but for sufficiently high incident power level it provides gain at shifted frequency.

Such nonlinearity sometimes is detrimental to long haul optical communication system.

\section{A. Non-return to zero(NRZ)}

\section{Modulation Format Overview}

In the NRZ format the pulse remains on throughout the bit slot and its amplitude does not drop to zero between two or more successive bits. As a result, pulse width varies depending on the bit pattern. In the early days or in commercial system NRZ are used in fiber-optical communication, due to a) it is not sensitive to laser phase noise b) it requires a relatively low electrical bandwidth for transmitters and receivers compared with RZ; c) it has the simplest configuration of transmitter and receiver; d)less cost. Unfortunately, NRZ modulation format is not appropriate for high bit rate and long distances optical communication system [6][8].NRZ modulation may be better in case of large number of channels.

\section{B. RETURN TO ZERO(RZ)}

In the RZ format each pulse that represents bit 1 is shorter than the bit slot, and its amplitude returns to zero before the bit duration is over. Pulse width remains the same in the case of RZ format. RZ is used in far away communication signals in which the signal drops to zero between each pulse. This takes place even if a number of consecutive "0" or " 1 " occurs in the signal. The RZ pulse code modulation signal is self-clocking [5]. Therefore, separate clock does not need to be sent alongside the signal, but suffers from using twice the bandwidth to obtain the same data-rate as compared to NRZ format. The main characteristic of RZ modulated signals is a relatively broad optical spectrum, resulting in a reduced dispersion tolerance and a reduced spectral efficiency [6].RZ pulse enables an increased fairness to fiber nonlinear effects [8].

\section{Simulation And Design Consideration}

Optical communication system consists of a transmitter, a transmission channel and receiver. Transmitter consists a continuous wave laser $(\mathrm{CW})$, pseudo random bit sequence generator(PRBS),pulse modulation generator and modulator. Single mode Optical fiber(SMF) is used as a transmission channel. Receiver side consists a photodetector.The input signal has been generated by non return to zero(NRZ) and return to zero(RZ) pseudorandom binary sequence in two simulation set up. Input signal is modulated with continuous wave laser (CW) through Mach-Zehnder modulator. Input signal is supplied by continuous laser with $1550 \mathrm{~nm}$ wavelength at power $5 \mathrm{dbm}$ which is vastly modulated at $10 \mathrm{Gbps}$. with different modulation 
format in Mach-Zehnder modulator. The extinction ratio 30db has been applied to Mach-zehnder modulator. In this optical communication system FBG and EDFA have been used to the purpose of dispersion compensation and signal amplification respectively. By try and error method [4] grating length is equal to 6mm.The Dispersion compensated signal passes through Erbium-doped fiber amplifier (EDFA). EDFA amplify the signal before receive by photo detector PIN. Then amplified signal will pass through Bessel optical filter. Properties of the Bessel optical filter are carrier wavelength \& lambda; $=1550 \mathrm{~nm}$ and bandwidth $=4 \mathrm{x}$ Bit rate. The initial setting for proposed design with a view to optical transmission take place is shown below.

Input power: $5 \mathrm{dbm}$

Frequency at CW laser: $1550 \mathrm{~nm}$

Fiber length: $25 \mathrm{~km}$

Attenuation Coefficient at cable section: $0.2 \mathrm{db} / \mathrm{km}$

Extinction ratio of Mach-zehnder modulator: $30 \mathrm{db}$

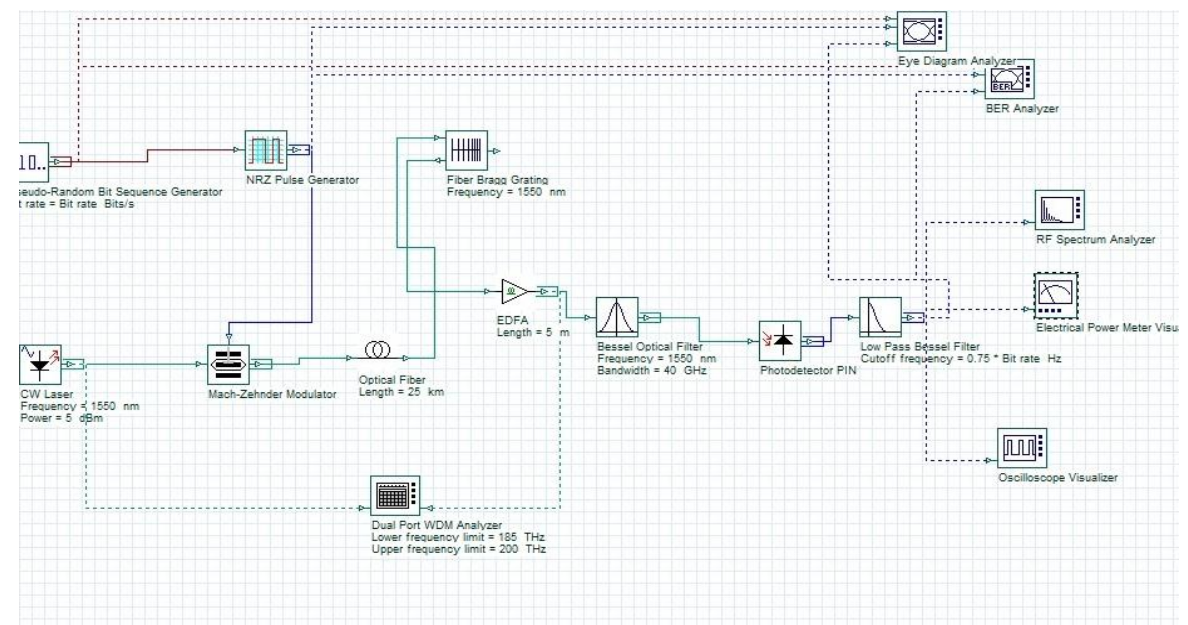

Fig.2 Design model of simulation system for NRZ.

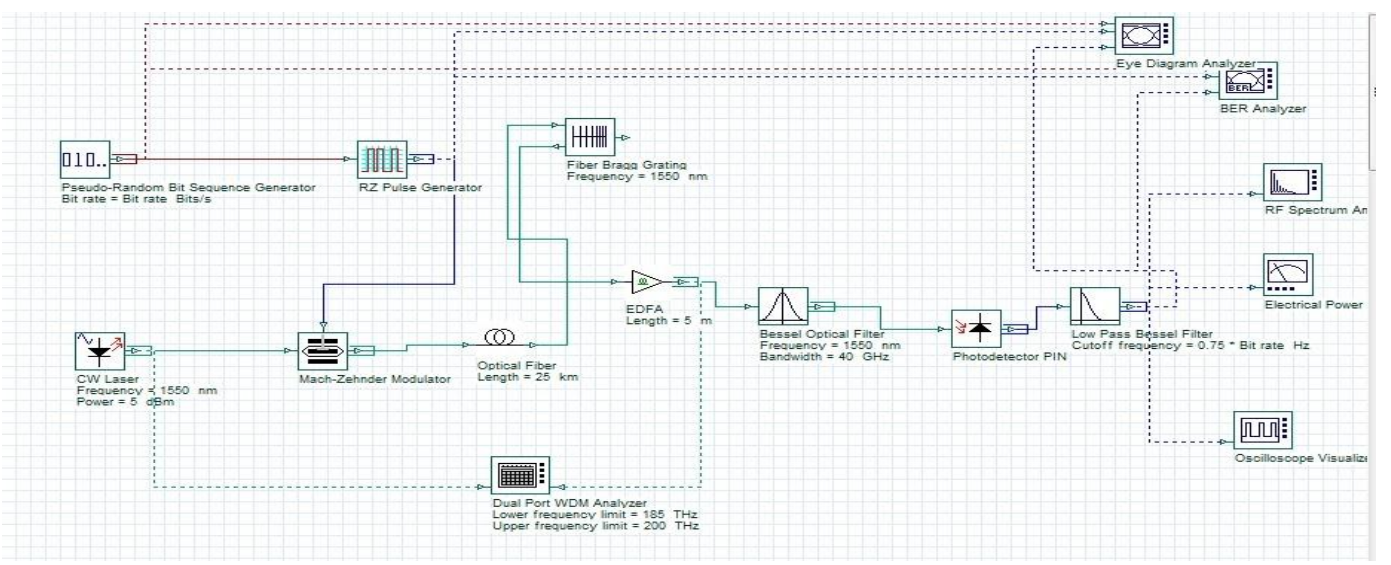

Fig.3 Design model of simulation system for RZ.

\section{Result And Discussion}

In this paper, analysis the parametric performance and comparison of modulation format in optical fiber transmission system is done by opti-system simulation tool. The outcome of experimental simulation at various transmissions distance using different modulation format for single transmission channel with dispersion compensator FBG and amplifier EDFA are tabulated in TABLE-1 and TABLE - 2 respectively. Amplification is performed with the help of the EDFA component and dispersion compensation with help of FBG. The eye diagrams are also shown in figure.4 (a) to 4(c). 

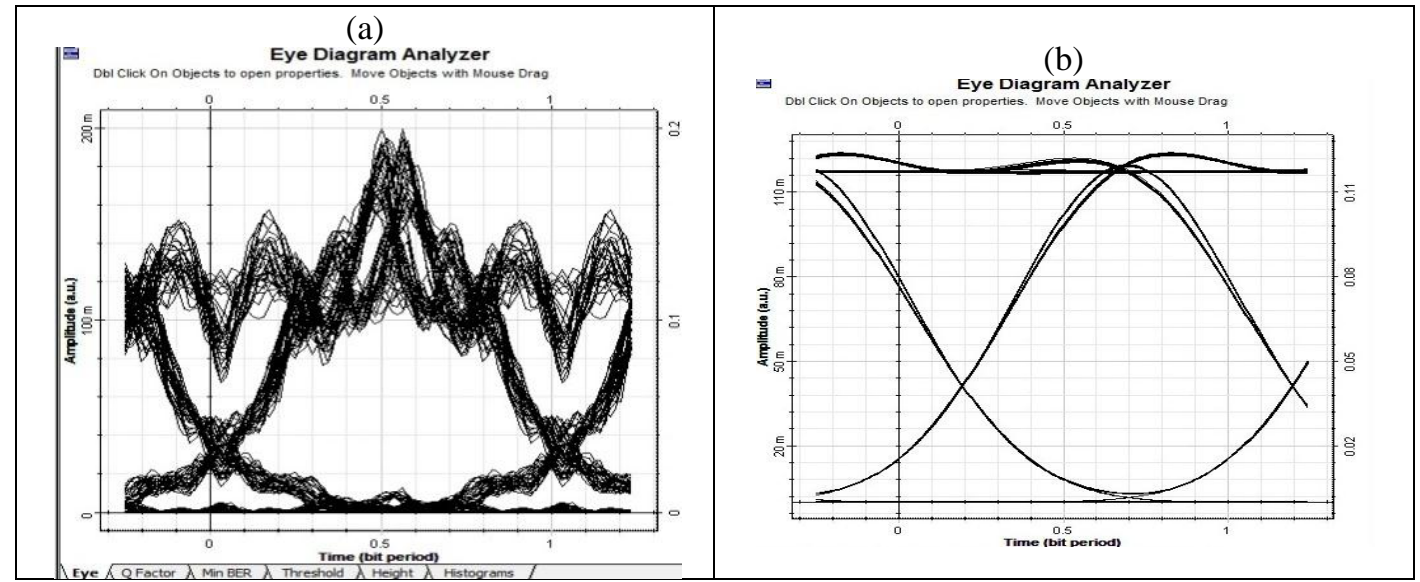

(c)

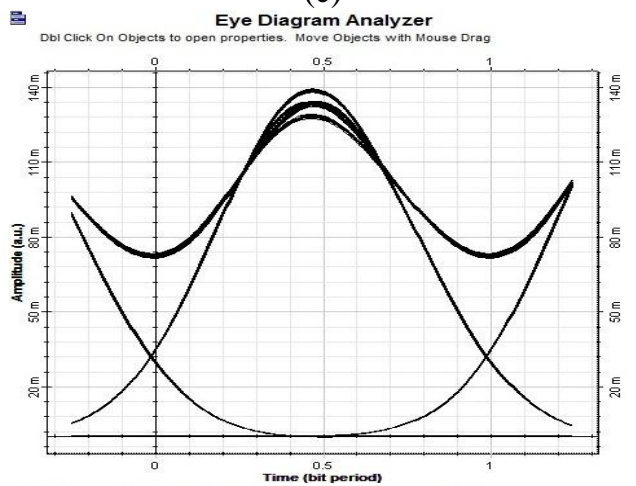

Fig. 4. (a) Eye diagram without FBG; (b) Eye diagram with NRZ ; (c) Eye diagram with RZ

Table 1: NRZ with FBG and EDFA (Performance with variation of length)

\begin{tabular}{|c|l|l|l|l|}
\hline $\begin{array}{c}\text { Length } \\
(\mathrm{km})\end{array}$ & $\begin{array}{l}\text { Gain } \\
(\mathrm{db})\end{array}$ & $\begin{array}{l}\text { Noise Figure } \\
(\mathrm{db})\end{array}$ & $\begin{array}{l}\text { Power } \\
(\mathrm{mw})\end{array}$ & Q-Factor \\
\hline 5 & 12.394657 & 9.4757294 & 5.916 & 136.377 \\
\hline 10 & 12.352340 & 10.411669 & 5.797 & 158.377 \\
\hline 15 & 12.308222 & 11.361412 & 5.673 & 139.311 \\
\hline 20 & 12.260640 & 12.323485 & 5.542 & 88.1212 \\
\hline 25 & 12.207873 & 13.296521 & 5.40 & 62.0838 \\
\hline
\end{tabular}

Table 2: RZ with FBG and EDFA (Performance with variation of length)

\begin{tabular}{|c|l|l|l|l|}
\hline $\begin{array}{c}\text { Length } \\
(\mathrm{km})\end{array}$ & $\begin{array}{l}\text { Gain } \\
(\mathrm{db})\end{array}$ & $\begin{array}{l}\text { Noise Figure } \\
(\mathrm{db})\end{array}$ & $\begin{array}{c}\text { Power } \\
(\mathrm{mw})\end{array}$ & Q-Factor \\
\hline 5 & 11.388796 & 13.440236 & 6.239 & 345.705 \\
\hline 10 & 11.340763 & 14.408657 & 5.980 & 217.964 \\
\hline 15 & 11.285926 & 15.384181 & 5.659 & 138.794 \\
\hline 20 & 11.220942 & 16.368030 & 5.295 & 95.8806 \\
\hline 25 & 11.141951 & 17.361920 & 4.907 & 61.7445 \\
\hline
\end{tabular}

It is clearly seen that in both RZ and NRZ modulation format by increasing fiber length the gain and power decreases but noise figure increases due to dispersion and attenuation, thus decreased the performance of the (Bit Error Rate) BER and power received respectively. 


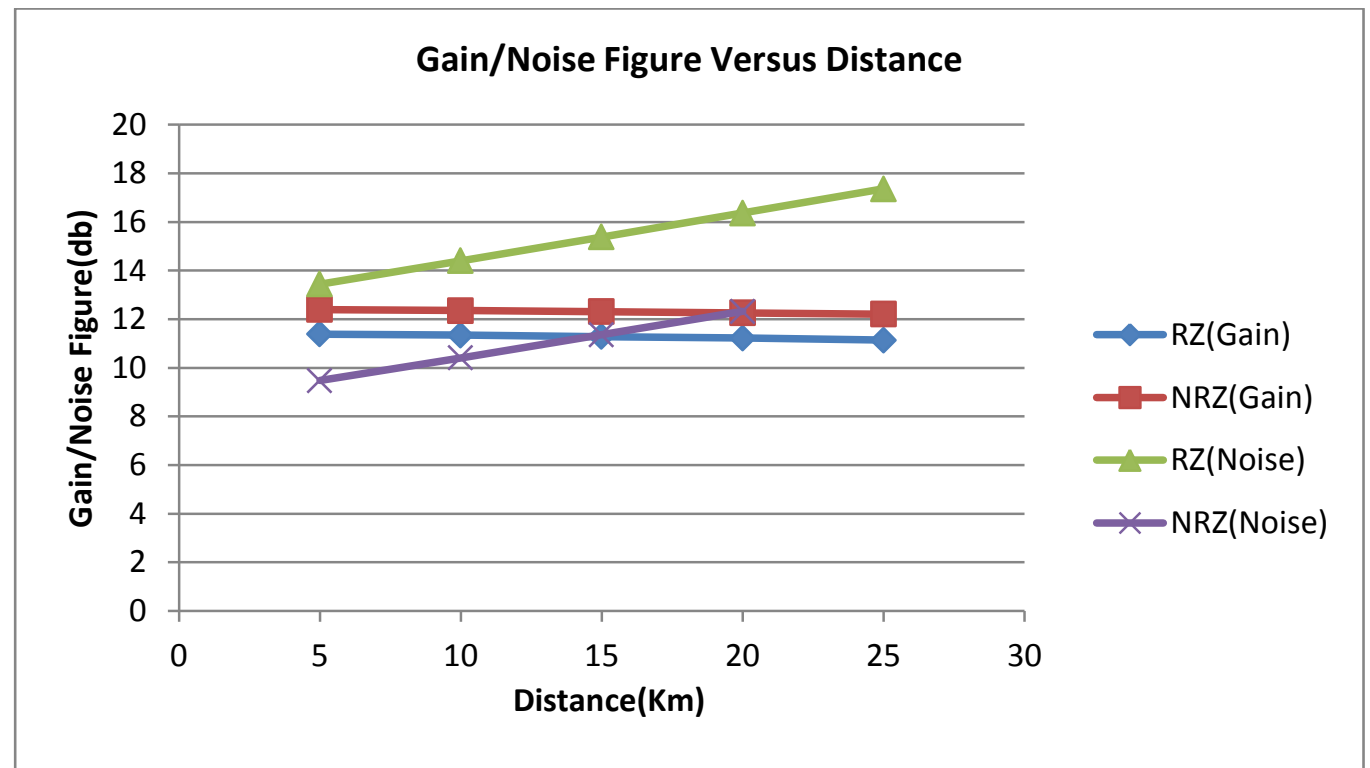

Fig. 5. Variation of gain/noise figure versus distance.

From fig. 5 it is observed that NRZ(gain) is high in comparison to RZ(gain) and NRZ(noise figure) is less than RZ(noise figure) It shows that NRZ gives better performance than RZ for single transmission channel with SMF at low bit rate and for short distance. We have focused on the Q- factor of RZ modulation and NRZ modulation and analyzed that overall RZ has greater Q-factor than NRZ with respect to various distances.

Table 3: NRZ modulation (Performance with variation of input power)

\begin{tabular}{|c|c|}
\hline Input Power(dbm) & Q-Factor \\
\hline 1 & 60.4391 \\
\hline 5 & 62.0838 \\
\hline 10 & 57.1126 \\
\hline 15 & 45.0770 \\
\hline 20 & 35.6482 \\
\hline
\end{tabular}

Table 4: RZ modulation (Performance with variation of input power)

\begin{tabular}{|c|c|}
\hline Input power(dbm) & Q-Factor \\
\hline 1 & 56.05531 \\
\hline 5 & 61.7445 \\
\hline 10 & 65.2898 \\
\hline 15 & 99.1628 \\
\hline 20 & 77.5717 \\
\hline
\end{tabular}

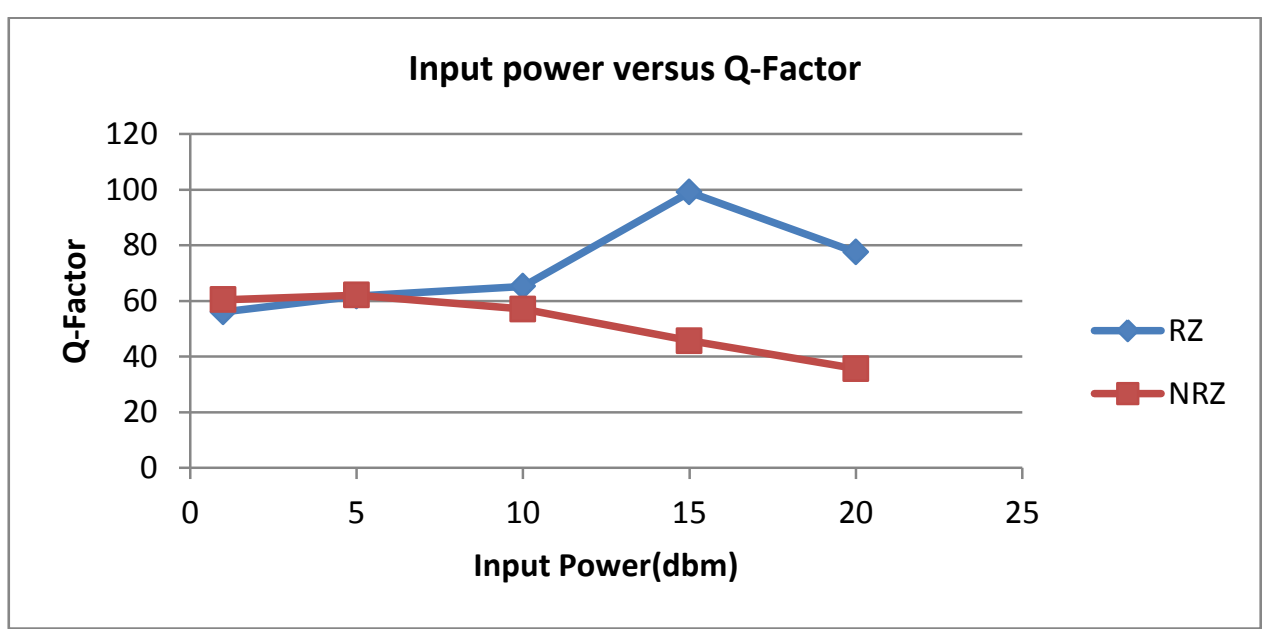

Fig. 6 Variation of Q factor versus input power. 


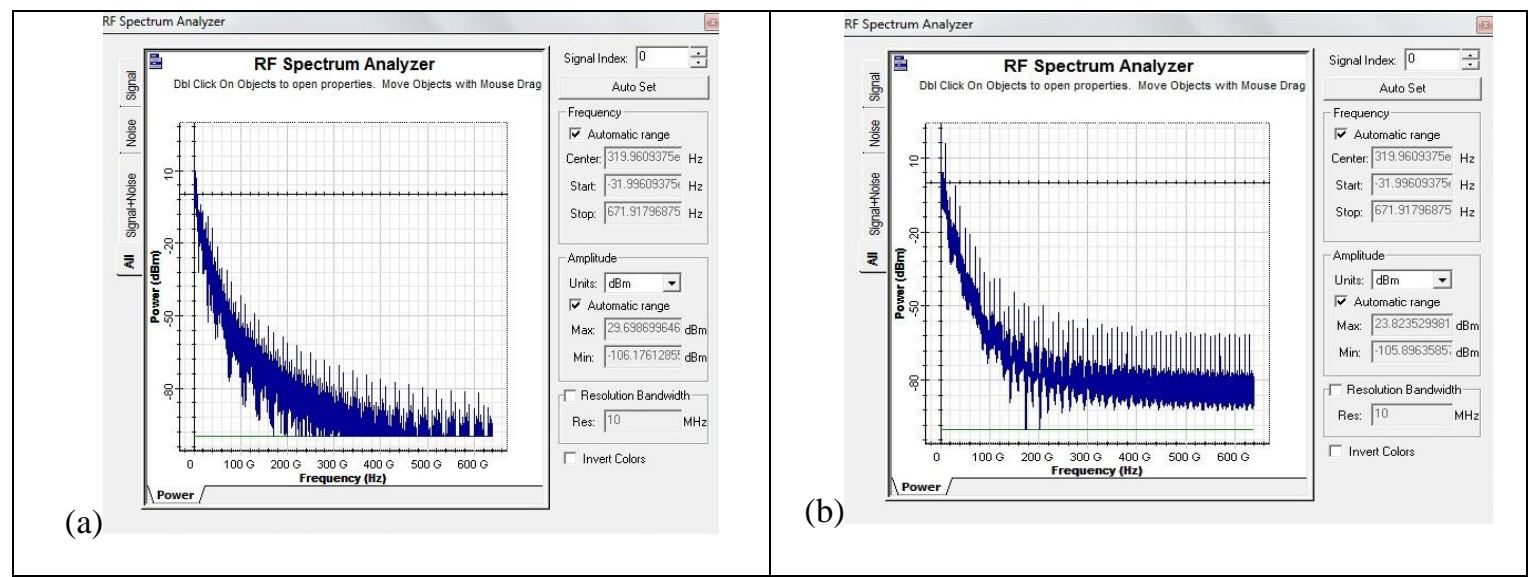

Fig. 7. (a) RF spectrum analyzer of NRZ;(b) RF spectrum analyzer of RZ.

Fig.6 shows that Q factor increases initially with launched power, reaches a peak value, and then decreases with a further increase in power because of the onset of the nonlinear effects. A moderate bigger value of laser average power is favourable to the performance of the transmission system. And from the figure we can find that in RZ with the input optical power increased to about $10 \mathrm{dBm}$, the Q factor increases. When the input optical power approaches $15 \mathrm{dBm}$, the $\mathrm{Q}$ factor becomes the maximum. When the input optical power is greater than $16 \mathrm{~dB}$ the quality factor decreased gradually and the error performance is gradually degraded. In NRZ modulation optical power approaches $5 \mathrm{dbm}$, the Q factor becomes maximum and after that Q factor gradually decreases and BER occurs in scenario. It is also estimated from graphs, that if power consumption is increased at the central office, it can transmit high data rates with minimum BER, and it also shows that if power consumption is lowered in the central office, it works for lower data rates with optimum BER.

We have also studied the spectral content of both NRZ and RZ data. Figure 7 shows the respective spectrums for both RZ and NRZ data streams. RZ data has higher spectral content compared to NRZ. Another observation is that a very strong clock spectral line exists in the RZ spectrum, whereas a spectral null occurs at the clock frequency for NRZ data. This makes clock recovery simpler when using RZ data

The performance of the NRZ, RZ has been observed which shows that RZ is better for long optical communication system at high data rate. Through the whole system study found that the performance of mix compensation system is best in the long-distance high-speed WDM systems. So the Q-Factor is very much acceptable for RZ system for high speed and long distance transmission in optical network.

\section{Conclusion}

In this paper we have visualized various graph and measurements on eye diagram, spectrum analyzer and following have been observed.

NRZ is better and is used for small distance communication system at low bit rates. An advantage of the NRZ format is that the bandwidth associated with the bit stream is smaller than that of the RZ format by about a factor of 2 simply because on-off transitions occur fewer times.

The RZ modulation format is best for the long distance optical communication system due to its low value of Jitter and BER at high bit rate with high input power.

\section{References}

[1] M.A Othman, M.M Ismail, H.A Sulaiman "An analysis of $10 \mathrm{Gbits} / \mathrm{s}$ optical transmission sytem using fiber bragg gratting.IOSRJEN.Vol.2,issue 7(July 2012).pp 55-61.

[2] M.Litchinitser,J.Eggleton, and B.Patterson, "Fiber Bragg Gratting for Dispersion Compensation in Transmission theoretical Model and Design Criteria for Nearly Ideal Pulse Recompression", Journal oflightwave Technology,Vol.15No.8,August 1997.

[3] Prachi Shukla, Kanwar Preet Kaur "Performance Analysis of EDFA for different Pumping Configurations at High Data Rate" International Journal of Engineering and Advanced Technology (IJEAT) ISSN: 2249 - 8958, Volume-2, Issue-5, June 2013.

[4] S.O Mohammadi,SaeedMozzaffari and M.Mahdi Shahidi, "Simulation of a transmission sytem to compensate dispersion in an optical fiber by chirp grating."International Journal of the Physical Sciences, Vol.6(32).pp7354-7360,2December,2011.

[5] MUTHANA Y. ALDOURI, S. A. ALJUNID, R. BADLISHAH AHMAD, HILAL A. FADHIL, "Bit error rate (BER) performance of return-to-zero and non return-to-zero data signals optical code division multiple access (OCDMA) system based on AND detection scheme in fiber-to-the-home (FTTH) networks". Optica Applicata, Vol. XLI, No. 1, 2011.

[6] A. García-Pérez, J. A. Andrade-Lucio, O. G. Ibarra-Manzano, E. Alvarado-Méndez,,M. Trejo Duran and H. Gutierrez Martin "Modulation Formats for High Bit-Rate Fiber Transmission". Vol. 16 no. 2 Mayo-Agosto 2006.

[7] Bo Xu, "Study of Fiber Nonlinear Effects on Fiber Optic Communication Systems". doctral diss., University of Virginia, august 2003.

[8] V. Bobrovs, J. Porins, G. Ivanovs "Influence of Nonlinear Optical Effects on the NRZ and RZ Modulation Signals in WDM Systems" ISSN 1392 - 1215 SIGNALU TECHNOLOGIJA 2007. Nr. 4(76) 